\title{
Plasmon Loss Spectroscopy of Au/ZnO nanoparticles: Prospects for Ultrafast Dynamics in Hybrid Nanostructures
}

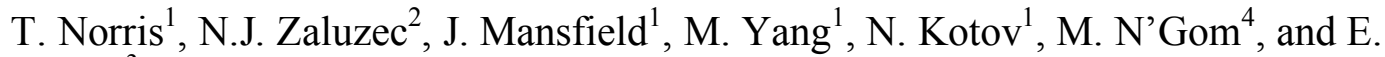 \\ Olsson $^{3}$ \\ ${ }^{1}$ Center for Ultrafast Optical Science, University of Michigan Ann Arbor, MI, USA \\ ${ }^{2}$ Electron Microscopy Center, Argonne National Laboratory, Argonne Il. USA ${ }^{3}$ Dept. of \\ Applied Physics, Chalmers University of Technology, Gothenburg, Sweden; ${ }^{4}$ Institute for \\ Defense Analyses, Alexandria, VA, USA.
}

Recent advances in TEM energy loss spectroscopy have enabled energy resolution as low as $0.2 \mathrm{eV}$. From the point of view of optics, this is a key advance as it enables for the first time high spatial resolution measurements of the optical-frequency resonances of nanoparticles. An enormous body of literature has been built using ultrafast optical techniques to study the physics of many types of nanoparticles, but even near-field optical techniques are only able to reach spatial resolutions of order $100 \mathrm{~nm}$, roughly three orders of magnitude worse than can be obtained in TEM. Thus the emerging field of coupled EELS and optics promises to provide a powerful new approach to the study of nanostructures at high spatial and temporal resolution.

To date, energy loss spectroscopy and imaging has been reported for metal nanoparticles, characterizing the optical plasmon modes of Ag and Au nanoparticles in the 2-4 eV energy range $[1,2]$. We have also investigated the modes of coupled Au nanorods using energy-filtered TEM, and demonstrated the connection between the energy-loss image and the electromagnetic field distribution calculated by the discrete-dipole approximation method [3]. Most recently, the first results on ultrafast EELS measurements on nanoplasmonic systems have been reported [4].

In this work, we present initial investigations into semiconductor nanoparticles and metal-semiconductor nanostructure hybrids. Specifically, we are studying opticalfrequency energy loss in structures consisting of Au nanospheres attached to $\mathrm{ZnO}$ triangular nanoparticles. The goal is to lay the foundation for studies of the physics of electronic coupling in novel hybrid nanostructures.

Both imaging and electron energy loss spectroscopy of the $\mathrm{Au} / \mathrm{ZnO}$ nanoparticles were carried out at 80, 120, and $300 \mathrm{kV}$ using a FEI Tecnai F20 (ANL) and a FEI 80-300 Titan (Chalmers). EELS on the Tecnai was conducted using a standard Schottky FEG, while the Titan was equipped with both a Cs probe corrector and a monochromator. Energy resolution on the Tecnai and on the Titan without the monochromator was typically $0.75 \mathrm{eV}$ while the Titan readily achieved $0.3 \mathrm{eV}$ with the monochromator. The first studies in this system concentrated upon finding conditions under which the specimen was stable and did not undergo radiation damage effects. An example of this damage is dramatically illustrated in Figure 1, which shows the severe mass loss of $\mathrm{ZnO}$ which occurs above $100 \mathrm{kV}$, while the Au nanoparticles are negligibly affected. In addition to mass loss, the $\mathrm{ZnO}$ undergoes major changes becoming almost metallic in nature. This can be seen by reference to the low loss structure of the $\mathrm{ZnO}$ in figure 2, which compares results measured at 300 and $80 \mathrm{kV}$. An oxide band gap is clearly visible at $80 \mathrm{kV}$, this is completely absent at higher voltages. The observed gap is around $3 \mathrm{eV}$, close to the expected value of $3.3 \mathrm{eV}$ from optical measurements. 


\section{References:}

1. J. Nelayah, et al., Nature Phys. 3, 348 (2007).

2. M. N'Gom, J. Ringnalda, J.F. Mansfield, A. Agarwal, N. Kotov, N.J. Zaluzec, and T.B. Norris, Nano Lett. 8, 3200 (2008).

3. M. N'Gom, S. Li, G. Schatz, R. Erni, A. Agarwal, N. Kotov, and T.B. Norris, Phys. Rev. B 80, 113411 (2009).

4. B. Barwick, D.J. Flannigan, and A. H. Zewail, Nature 462, 902 (2009).

5. This work was supported in part by the US DOE Office of Science, Contract DEAC02-06CH11357 at ANL

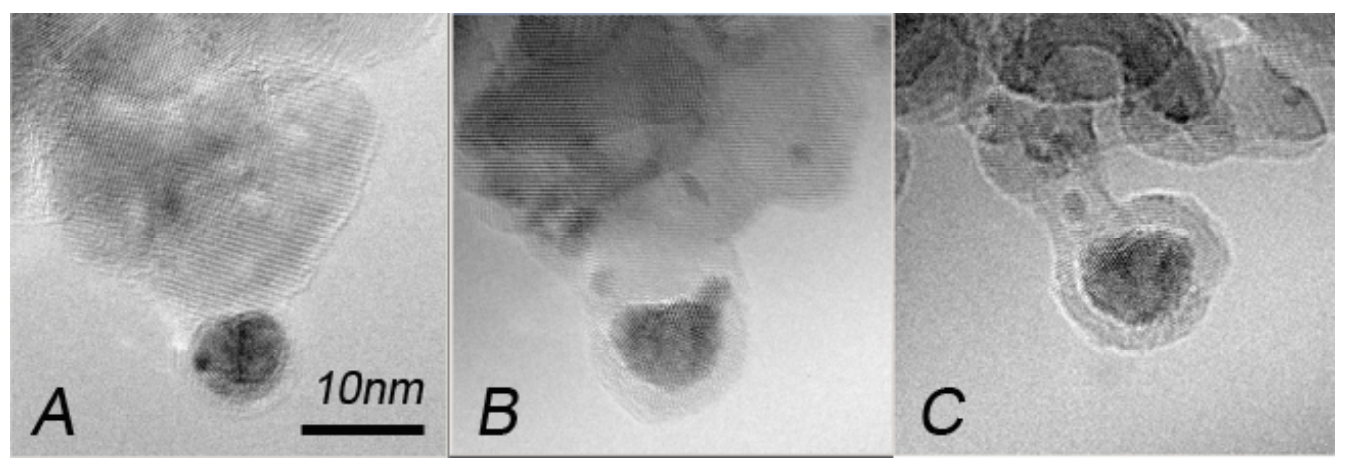

Figure 1. ) Time evolution images of a $\mathrm{Au}$ sphere and $\mathrm{ZnO}$ triangular nanoparticles under $300 \mathrm{kV}$ electron irradiation. Note the severe mass loss of the $\mathrm{ZnO}$.
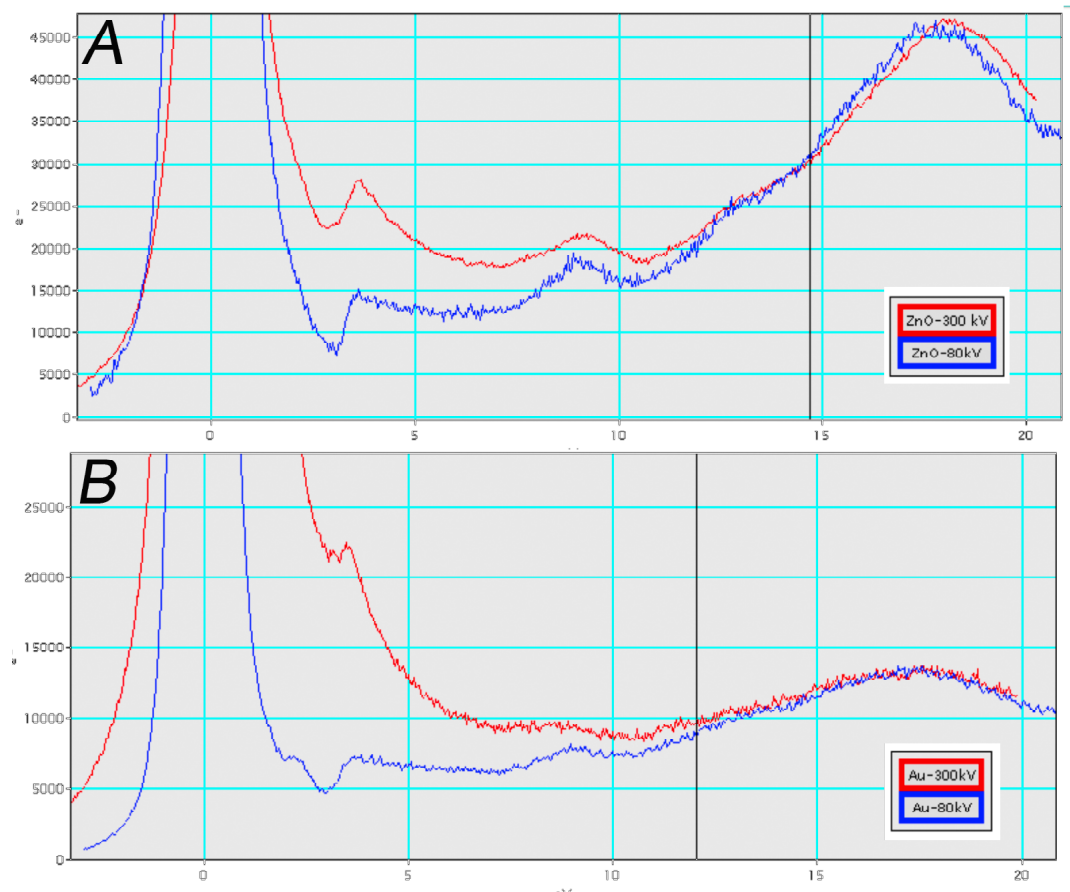

Figure 2.) Electron energy loss spectra from $\mathrm{ZnO}(\mathrm{A})$ and $\mathrm{Au}(\mathrm{B})$ nanoparticles measured at 80 (blue) and $300 \mathrm{kV}$ (red). Notice the small but detectable peak in the Au spectra at $2.6 \mathrm{eV}$ which is not present in the $\mathrm{ZnO}$ spectra. The Au NP has a "coating" layer on it in all spectra that is principally $\mathrm{ZnO}$. 